\title{
A Phase II study of Bendamustine in Combination With Rituximab as Initial Treatment for Patients With Indolent non-follicular non-Hodgkin's Lymphoma.
}

Stefano Luminari, Maria Goldaniga, Marina Cesaretti, Lorella Orsucci, Alessandra Tucci, Alessandro Pulsoni, Flavia Salvi, Luca Arcaini, Angelo Michele Carella, Alessandra Tedeschi, Antonello Pinto, Caterina Stelitano \& Luca Baldini

To cite this article: Stefano Luminari, Maria Goldaniga, Marina Cesaretti, Lorella Orsucci, Alessandra Tucci, Alessandro Pulsoni, Flavia Salvi, Luca Arcaini, Angelo Michele Carella, Alessandra Tedeschi, Antonello Pinto, Caterina Stelitano \& Luca Baldini (2015): A Phase II study of Bendamustine in Combination With Rituximab as Initial Treatment for Patients With Indolent non-follicular non-Hodgkin's Lymphoma., Leukemia \& Lymphoma, DOI: 10.3109/10428194.2015.1091934

To link to this article: http://dx.doi.org/10.3109/10428194.2015.1091934

View supplementary material $₫$

曲 Accepted online: 17 Sep 2015.

Submit your article to this journal $2 \pi$

Џ Article views: 10

View related articles $\nearrow$

View Crossmark data $\nearrow$ 


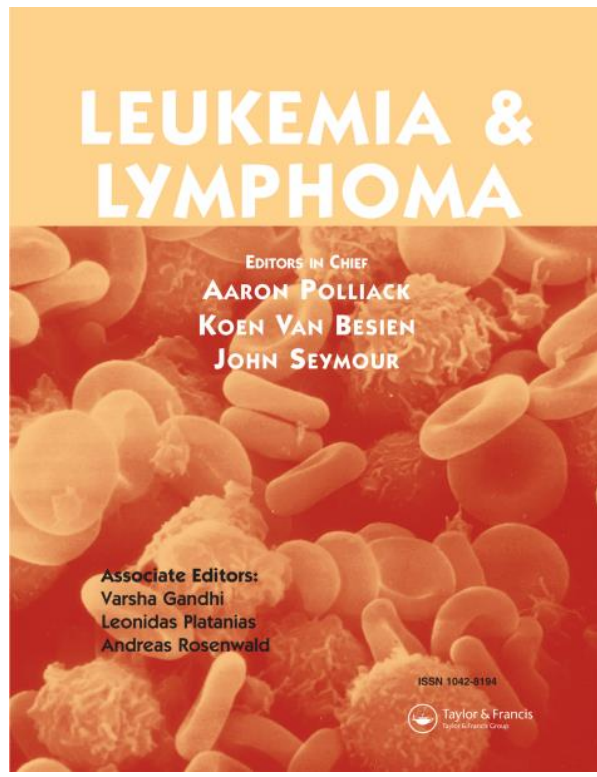

$86 \%$ (IC 75.0-92.8).
A Phase II study of Bendamustine in Combination With Rituximab as Initial Treatment for Patients With Indolent non-follicular nonHodgkin's Lymphoma.

Stefano Luminari, Maria Goldaniga, Marina Cesaretti, Lorella Orsucci, Alessandra Tucci, Alessandro Pulsoni, Flavia Salvi, Luca Arcaini, Angelo Michele Carella, Alessandra Tedeschi, Antonello Pinto, Caterina Stelitano and Luca Baldini

doi: 10.3109/10428194.2015.1091934

\section{Abstract}

The purpose of this phase 2 study was to determine the activity and safety of 6 cycles of bendamustine and 8 rituximab (RB) as first-line treatment of adult patients with advanced stage non-follicular indolent non-Hodgkin lymphomas (INFL). The primary endpoint was the complete response rate (CRR) with expected CRR of $75 \%$.

Sixty-nine patients were enrolled; median age was 65 years (45-75), 65\% were male, $93 \%$ of patients had stage IV disease.

Complete and overall response rates were $48 \%$ (95\% IC 35.6-60.2), and

The most common grade $3 / 4$ adverse events were neutropenia (43\%), thrombocytopenia (7\%), anemia (4\%); whereas the rate of febrile neutropenia was very low (3\%). At a median follow up of 22 months (1-43 months), 2-year progression free survival was $89 \%$ (IC: 79-95), and 2-year overall survival was $96 \%$ (IC: $87-99$ ).

RB combination is active and well tolerated in patients with advanced stage previously untreated INFL.

(C) 2015 Taylor \& Francis. This provisional PDF corresponds to the article as it appeared upon acceptance. Fully formatted PDF and full text (HTML) versions will be made available soon.

DISCLAIMER: The ideas and opinions expressed in the journal's Just Accepted articles do not necessarily reflect those of Taylor \& Francis (the Publisher), the Editors or the journal. The Publisher does not assume any responsibility for any injury and/or damage to persons or property arising from or related to any use of the material contained in these articles. The reader is advised to check the appropriate medical literature and the product information currently provided by the manufacturer of each drug to be administered to verify the dosages, the method and duration of administration, and contraindications. It is the responsibility of the treating physician or other health care professional, relying on his or her independent experience and knowledge of the patient, to determine drug dosages and the best treatment for the patient. Just Accepted articles have undergone full scientific review but none of the additional editorial preparation, such as copyediting, typesetting, and proofreading, as have articles published in the traditional manner. There may, therefore, be errors in Just Accepted articles that will be corrected in the final print and final online version of the article. Any use of the Just Accepted articles is subject to the express understanding that the papers have not yet gone through the full quality control process prior to publication. 


\title{
A Phase II study of Bendamustine in Combination With Rituximab as Initial Treatment for Patients With Indolent non-follicular non-Hodgkin's Lymphoma.
}

\begin{abstract}
Authors:
Stefano Luminari ${ }^{1}$, Maria Goldaniga ${ }^{2}$, Marina Cesaretti ${ }^{1}$, Lorella Orsucci ${ }^{3}$, Alessandra Tucci ${ }^{4}$, Alessandro Pulsoni ${ }^{5}$, Flavia Salvi ${ }^{6}$, Luca Arcaini ${ }^{7}$, Angelo Michele Carella ${ }^{8}$, Alessandra Tedeschi $^{9}$, Antonello Pinto ${ }^{10}$, Caterina Stelitano ${ }^{11}$ and Luca Baldini ${ }^{2}$.
\end{abstract}

\section{Institutions:}

${ }^{1}$ Department of Diagnostic, Clinical, and Public Health Medicine, University of Modena and Reggio Emilia, Modena, Italy;

${ }^{2}$ Fondazione Ospedale Maggiore Policlinico, Mangiagalli e Regina Elena, Milano;

${ }^{3}$ Hematology, Città della Salute e della Scienza, Torino, Italy;

${ }^{4}$ Division of Hematology, Spedali Civili, Brescia, Italy;

${ }^{5}$ Division of Hematology, Sapienza University, Roma, Italy;

${ }^{6}$ Azienda ospedaliera nazionale SS. Antonio e Biagio e Cesare Arrigo, Alessandria;

${ }^{7}$ Department of Hematology Oncology, Fondazione IRCCS Policlinico San Matteo, University of Pavia, Pavia, Italy;

${ }^{8}$ Azienda Ospedaliera Universitaria 'San Martino', Genova;

${ }^{9}$ Azienda Ospedaliera Ospedale Niguarda Ca' Granda, Milano;

${ }^{10}$ UOSC di Ematologia Oncologica, Istituto Nazionale Tumori, Fondazione Pascale, IRCCS, Napoli;

${ }^{11}$ Azienda ospedaliera Bianchi Melacrino Morelli, Reggio Calabria; 


\section{Corresponding Author:}

Stefano Luminari, MD, Department of Diagnostic, Clinical and Public Health Medicine, University of Modena and Reggio Emilia - Via del Pozzo 71, 41124 Modena, Italy. Phone +39-059-4223286; Fax +39-059-4223707; e-mail: stefano.luminari@ unimore.it

\section{Abstract word count: 150}

Text word count: 3786

Number of tables: 3

Number of Figures: 2

Number of references: 23

Running head: Bendamustine and rituximab for INFL.

Keywords: Indolent non-follicular lymphomas, chemotherapy, bendamustine, rituximab. 
ABSTRACT

The purpose of this phase 2 study was to determine the activity and safety of 6 cycles of bendamustine and 8 rituximab (RB) as first-line treatment of adult patients with advanced stage non-follicular indolent non-Hodgkin lymphomas (INFL). The primary endpoint was the complete response rate (CRR) with expected CRR of $75 \%$.

Sixty-nine patients were enrolled; median age was 65 years (45-75), $65 \%$ were male, $93 \%$ of patients had stage IV disease.

Complete and overall response rates were $48 \%$ (95\% IC 35.6-60.2), and 86\% (IC 75.0-92.8). 
The most common grade $3 / 4$ adverse events were neutropenia (43\%), thrombocytopenia (7\%), anemia (4\%); whereas the rate of febrile neutropenia was very low (3\%). At a median follow up of 22 months (1-43 months), 2-year progression free survival was $89 \%$ (IC: 79-95), and 2-year overall survival was 96\% (IC: 87-99).

RB combination is active and well tolerated in patients with advanced stage previously untreated INFL.

INTRODUCTION

Non-follicular indolent non-Hodgkin lymphomas (INFLs) are mature B-cell neoplasms that encompass a heterogeneous group of lymphoid malignancies, including small lymphocytic 
(SLL), lymphoplasmacytic (LPL/IC), and marginal zone lymphomas (MZL) [1]. INFLs are slowgrowing tumors that typically affect the older population and are characterized by a prolonged natural history with a median survival of up to 10 years [1]. Standard treatment does not differ among INFL subtypes and ranges from a "wait and see" approach for patients with low tumor burden to systemic chemotherapy for symptomatic patients [2-9]. As in follicular cell lymphomas or chronic lymphocytic leukemia (CLL), chemoimmunotherapy combining rituximab with alkylating agents or purine analogues represents the most widely accepted diffuse treatment option [10-12].

The Fondazione Italiana Linfomi (FIL, former "Intergruppo Italiano Linfomi") conducted several studies on INFL to assess the role of anthracyclines in a randomized phase III trial [11] and the activity of fludarabine in combination with cyclophosphamide (FC) in a phase II study [13]. More recently, the activity of FC combined with rituximab (FCR) followed by rituximab maintenance was investigated in a further trial [12]. This study demonstrated that FCR was very active in INFL, with an overall response rate (ORR) of $89.1 \%$, a complete remission rate (CRR) of $67.4 \%$ and a substantial 3-year progression-free survival (PFS) of 90.1\%. Despite its impressive antitumor efficacy, the acute toxicity profile of the FCR combination as well as the risk of secondary malignancies associated with fludarabine-based regimens represent major concerns [12], stimulating the search for safer and equally active regimens.

Recently, bendamustine was shown to be a very active agent in B-cell tumors and when combined with rituximab $(R)$ as a front line treatment of indolent lymphomas, was resulting at least as active but by far less toxic than R-CHOP [14,15]. In 2011 FIL, launched the INFL09 
phase II trial, aimed at assessing activity and safety of rituximab and bendamustine (RB) combination as upfront therapy for patients with advanced INFLs.

\section{DESIGN AND METHODS}

\section{Study design}

We designed a prospective, multicenter, open-label, single-arm, phase II trial aimed to determine the activity and safety of a chemoimmunotherapy with 6 courses of bendamustine in combination with 8 doses of rituximab in patients with advanced untreated INFLs (INFL09, EudraCT number: 2010-019248-37, ClinicalTrials.gov Id: NCT01929265). The primary study endpoint was the CRR and the secondary endpoints were the partial response (PR) rate, ORR, safety, overall survival (OS) and PFS.

The study was conducted in 20 centers in Italy between February 2011 and March 2012. The study protocol was approved by the ethics committee of each institution. All patients gave written informed consent before treatment start. Data were collected using the Openclinica software (Community edition ver 3.1.2; Openclinica LLC, USA).

\section{Eligibility criteria}

Patients aged 18-75 years diagnosed with INFL (including SLL, LPL/IC, and MZL with the exclusion of splenic and primary extranodal MZL) according to the World Health Organization (WHO) classification of tumors of hematopoietic and lymphoid tissue [1], as demonstrated by lymph nodes and/or bone marrow biopsy, were included. Patient inclusion was based on local immunopathology assessment report as no upfront histology review was 
planned. Patients with histologic features consistent with MZL with concomitant involvement of the marrow, and/or spleen, and/or lymph nodes and/or extranodal sites but lacking the diagnostic features of splenic, nodal or extranodal MZL sites were categorized as disseminated MZL.

Additional inclusion criteria were: no previous treatment for lymphoma, stage III-IV disease or stage II disease with more than three involved sites, presence of at least one criteria for the definition of active disease (including systemic symptoms, hemoglobin $<10 \mathrm{~g} / \mathrm{dL}$, platelets $<100 \times 10^{9} / L$, diffuse bone marrow infiltrate, lymphocyte doubling time $<12$ months in leukemic cases, bulky disease $>7 \mathrm{~cm}$ ). Patients had to have adequate hepatic, renal, and cardiac function (left ventricular ejection fraction (LVEF) $\geq 45 \%$ at bidimensional echocardiogram), absolute neutrophil count (ANC) $\geq 1 \times 10^{9} / /$ and platelet count $\geq 75 \times 10^{9} /$, unless due to bone marrow involvement by lymphoma; an Eastern Cooperative Oncology Group (ECOG) performance status $\leq 2$ and a life expectancy $>6$ months.

\section{Study treatment}

Eligible patients were treated with 6 courses of RB combination, followed by 2 rituximab doses. Each cycle was administered every 28 days and consisted of rituximab $375 \mathrm{mg} / \mathrm{m}^{2}$ i.v. on day 1 (cycle 1 to 8), and bendamustine $90 \mathrm{mg} / \mathrm{m}^{2}$ i.v. on days $1-2$ or 2-3 (cycle 1 to 6 ) according to institutional/patient/physician choice. During cycle 1 rituximab could be administered on day 8 to prevent tumor lysis syndrome. At the end of the fourth RB course, patients were evaluated for tumor response; patients with progressive disease (PD) were considered as treatment failure, study protocol had to be discontinued, and adequate salvage therapy was administered according to local practice. Patients without evidence of disease progression completed the planned treatment. One month after administration of 
the last rituximab dose, patients had to be evaluated for tumor response. The delivered dose intensity (DI) was calculated according to Hryniuk, taking into account also rituximab [16].

During the follow-up phase, patients were evaluated for tumor response at months $6,12,18$ and 24.

Concomitant medications for supportive care and for medical conditions other than lymphoma were permitted, as clinically indicated. Cotrimoxazole and antifungal prophylaxis was mandatory. Antiviral prophylaxis including acyclovir was allowed but not mandatory.. For $\mathrm{HBCAb}$ positive patients, prophylaxis for hepatitis B reactivation with lamivudine 100 $\mathrm{mg} /$ die was mandatory from the start of the treatment until one year after the end of the treatment.

\section{Efficacy assessments}

Tumor response was defined according to the 2007 revised version of Cheson's criteria [17]. In case of initial bone marrow involvement, the procedure had to be repeated at the end of treatment; if not done patients could not be defined as complete remission (CR). Response was assessed locally and centrally reviewed blinded of patients outcome.

\section{Survival and toxicity}

The OS was defined as the time from study entry to the last observation or death from any cause. Patients who have not died at the time of the final analysis were censored at the date of the last contact. 
The PFS was defined as the time elapsed from study entry to the time of documented disease progression, relapse, or death from any cause. Responding patients, patients who are lost to follow up, those who withdraw the consent or drop-out due to adverse event were censored at the date of last available assessment. Toxicity was graded according to the National Cancer Institute Common Terminology Criteria for Adverse Events ( $\mathrm{NCl}$ CTCAE version 3.0, http://ctep.cancer.gov/reporting/ctc.html) and reported on a patient basis.

\section{Statistical analysis}

The safety analysis included all enrolled patients who received at least one dose of study agent and efficacy analysis was conducted on all eligible patients as per intention to treat principle.

The primary objective of the study was to demonstrate clinical activity of RB regimen in terms of CR rate. Initial study assumptions were based on response rates observed in indolent lymphomas in previous studies $[13,12]$. The median expected CR rate for advanced stage INFLs with the same characteristics treated with standard rituximab plus chemotherapy was estimated to be $60 \%(\mathrm{p} 0)$. The INFL09 study was designed with the hypothesis to increase CR rate to $75 \%$ (p1). Considering a two-tailed statistical test with an alpha error of 0.05 and a statistical power of $80 \%, 67$ patients were required at the end of the study and at least 47 CRs observed to confirm the initial hypothesis. Considering a dropout of $10 \%$, the final accrual was planned at 69 cases. The study was initially designed with a Simon's 2 stage design requiring at least 18 CRs among the first 27 treated patients.

RESULTS 


\section{Patient characteristics}

Between February 2011 and March 2012, 72 patients with INFLs were consecutively enrolled at 20 Italian institutions. Three patients were subsequently excluded due to major violation of inclusion criteria (one patient with mantle cell lymphoma and one with follicular lymphoma (FL) following local histology review) or to consent withdrawal (one patient). The main clinical and hematological characteristics of the 69 accrued patients are summarized in Table I. The main reasons for starting treatment were diffuse bone marrow involvement (38\%), hemoglobin less than $10 \mathrm{~g} / \mathrm{dL}(33 \%)$ and systemic symptoms or rapid disease progression (22\%). According to local pathology assessment, patients had LPL/IC in 32 cases, SLL in 17 and MZL in 20 cases. Pathology reports were subsequently reviewed with available clinical data being accurate enough to confirm the initial diagnosis in 54 (78\%) cases (26 LPL/IC, $12 \mathrm{SLL}, 16 \mathrm{MZL})$; unconfirmed cases were reclassified as SLL (1 case), as MZL (2 cases) and as LPL/IC (4 cases) or reported as low grade B cell lymphoma not otherwise specified (LG NOS) (8 cases) being cases with inconclusive immunohistochemistry details. Among the 18 patients with MZL, 6 cases were classified as nodal MZL and the remaining cases had the features of disseminated MZL.

Overall no HCV positive cases were enrolled; one and 7 patients were included with baseline HBsAg and anti-HBcAb positivity, respectively, and were all being treated with lamivudine prophylaxis.

Fifty-three out of 69 patients received all 6 planned courses of RB followed by the 2 R doses (76.8\%) and 59 at least 6 treatment cycles (85,5\%). Treatment was prematurely interrupted in 3 patients due to progressive disease (one after cycle 2, one after cycle 4 and one after cycle 6), in 6 due to toxicity (one after cycle 1, one after cycle 2, one after cycle 3 and three 
after cycle 4) and 2 due to the discovery of a second malignancy (one after cycle 1 and one after cycle 2) (Figure 1).

Overall, 487 RB cycles were administered during the study. All but 72 cycles were administered on time: median delay was 5 days (range 1 to 42). Rituximab was administered as planned in all but 9 cases for whom the antibody was started after the first cycle ( 3 patients) or prematurely discontinued due to adverse reaction (6 patients). Dose reductions were adopted in 5 patients and in 14 cycles (3\%). Bendamustine was administered as planned in all cases; dose reductions of bendamustine were prompted in 9 patients and in 18 cycles (4\%). Despite reported dose reductions, calculated DI was very high being 0.931 and 0.905 for bendamustine and rituximab respectively.

\section{$\underline{\text { Response assessment }}$}

The study was initially designed with a Simon's 2 stage design. However due to the very fast accrual we were not able to stop accrual at the planned stage I. Then due to the absence of safety issues observed with RB combination it was decided to perform a unique assessment of study endpoints with completion of study accrual (69 patients).

Based on the intention to treat analysis and on the local assessment of response, 39 patients achieved CR at the end of therapy (CRR=57\%; 95\% IC 44-68), and 59 patients had an objective response (ORR=86\%; 95\% IC 75.0-92.8). After centralized review 6 CR patients were reclassified as PR due to persistence of serum monoclonal component. Revised CR and ORR rates according to Cheson 2007 criteria [17] were 48\% (95\% IC 35.6-60.2) and 86\% (95\% IC 75.0-92.8), respectively (Table II). 


\section{Analysis of survival}

The median follow up at the time of current analysis was 22 months (range 1-43 months). Regarding the definition of PFS, 11 failures were recorded including 7 progressions, 3 relapses and 1 death for severe infection in a patient in CR.

As per OS, 3 patients died, two for progressive disease after 4 and 9 months from enrollment and one due to a severe infection after 3 months from enrollment.

On the basis of the ITT analysis, 2-years PFS was $89 \%$ (95\% Cl: $79-95)$, and OS was $96 \%$ (95\% Cl: 87-99) (Figure 2).

\section{$\underline{\text { Toxicity }}$}

The safety analysis was available for all 69 patients and for 487 cycles. Overall, the combination of RB resulted manageable and well tolerated.

The recorded toxicity was mainly restricted to the hematopoietic system. Thirty patients (43\%) experienced anemia that was graded as severe (Grade $\geq 3$ ) in 3 of them (4\%). Neutropenia was observed in 44 patients (63\%), of whom $30(43 \%)$ developed a grade $3 / 4$ event. Grade 3/4 febrile neutropenia occurred in 2 patients (3\%). Thrombocytopenia was observed in 21 patients (30\%), and was graded as severe in 5 (7\%); moreover in 5 patients thrombocytopenia was present before treatment start whereas only 1 patient developed severe thrombocytopenia during therapy (cycle 8).

Granulocyte stimulating factors (filgrastim or PEG filgrastim) were administered in 44 patients (64\%) and in 152 cycles (31\%). Use of G-CSF was described in $17 \%, 28 \%, 40 \%, 48 \%$, $45 \%$ and $41 \%$ of patients from cycle 1 to 6 and dropped to $17 \%$ and $11 \%$ at cycle 7 and 8 ; 
the median number of G-CSF doses calculated for filgrastim only was 4 (range 2 to 15). Erythropoietin was administered in 17 patients (25\%) and 40 cycles (8\%).

A detailed summary of hematological and non hematological events is reported in Table III.

One patient died during treatment due to a severe infection (fungal pneumonitis) after cycle 1. Three cases of secondary malignancy occurred: 1 Merkel skin tumor was diagnosed at cycle 1, 1 prostate cancer and 1 myofibroblastic tumor, both were diagnosed at cycle 2 .

\section{$\underline{\text { Subgroup analysis }}$}

Study results were also evaluated by patients subgroups as unplanned analysis. Comparable CR and ORR rates were observed among different histologic subtypes; CR was $56 \%, 43 \%$ and $62 \%$ in MZL, LPL/IC and SLL subtypes, respectively, without statistically significant differences (Table II); ORR was 72\%, 96\% and 92\% in MZL, LPL/IC, SLL with lower rates observed for MZL cases ( $P=0,017)$. Two-year PFS was 82\% (95\% IC 55-94), 97\% (95\% IC 7999) and 91\% (95\% IC 51-99) in MZL, LPL/IC and SLL subtypes, respectively, again with an inferior PFS observed for MZL cases $(p=0.0061)$.

\section{DISCUSSION}

The results of the INFL09 phase II trial demonstrated that bendamustine in combination with rituximab is an active and well tolerated regimen for patients with advanced stage symptomatic and untreated INFLs. Unfortunately, with the observed CR rates of $48 \%$ we missed our ambitious primary aim to show a $75 \%$ CR rate; however, considering the $86 \%$ ORR and the 2-year PFS of $89 \%$, our results are in line with those from other studies 
investigating the RB combination or different fludarabine-based regimens $[12,13]$. In terms of safety, RB was associated with a $43 \%$ of grade $3 / 4$ neutropenia with negligible rates of febrile neutropenia and infectious events, so confirming the favorable toxicity profile previously reported for this combination [18].

Since introduction of bendamustine, the RB combination has progressively been imposed as a standard approach for indolent non Hodgkin lymphoma and as an excellent alternative to other existing regimens. Differently from $\mathrm{CLL}$ and $\mathrm{FL}$, randomized trials supporting this choice in INFL are missing and treatment decisions for this subset of patients mainly rely on results of phase II trials or are extrapolated from results achieved in phase III studies also including other indolent lymphomas.

Before availability of bendamustine, initial treatment of patients with INFL was mainly based on fludarabine- or alkylator-based regimens [3]. This approach changed after the introduction of rituximab that was added to chemotherapy to boost anti-lymphoma activity of the various chemotherapy platforms [12]. As observed for CLL [19], excellent results were achieved with the FCR immunochemotherapy that was also studied by our group in a phase II study on 46 patients with INFL. By taking into account previous treatment outcomes for INFL, introduction of the FCR combination allowed a CR rate increase to $67.4 \%$ as compared to $29.4 \%$ and $40.6 \%$, usually achievable with alkylating agents or FC regimens, respectively $[11,13]$.

Looking at the best results obtained with FCR combination and despite a formal comparison is not appropriate, current data from the INFL09 failed to suggest a superiority of RB but can be used to support that the RB regimen has a similar anti-lymphoma activity to FCR. 
In addition, patients treated with fludarabine-based regimens displayed substantial rates of hematologic adverse events, mainly severe neutropenia and/or febrile neutropenia as well as infectious complications. These patients were also shown to be at significant risk of developing secondary malignancies, including myelodysplastic syndromes or acute leukemia, and solid tumors [20]. Furthermore, use of fludarabine has been acknowledged as a predictor for poor stem cell mobilization [21].

The INFL09 was specifically designed to study RB combination in INFL and results should be compared with those from other studies investigating the same combination in indolent lymphomas. In the StiL group trial [14], patients with previously untreated indolent and mantle cell lymphoma were randomized to RB versus R-CHOP. At median follow-up of 45 months median PFS was significantly longer in the RB group than in the R-CHOP group $(69,5$ months versus 31,2 months; $p<0,0001$ ) and $\mathrm{RB}$ was better tolerated than $\mathrm{R}-\mathrm{CHOP}$. The $\mathrm{ORR}$ did not differ between the treatment groups; however, the rate of CR was significantly increased in patients in the RB group ( $40 \%$ versus $30 \% ; p=0,021)$. Although the most frequent histotype for the Stil trial was FL, other indolent lymphoma subtypes were enrolled, including MZL (13\%), LPL/IC (8\%) and SLL (4\%). RB activity was similar across all histological subgroups with the only exception of MZL for which both RB and R-CHOP resulted in a similar median PFS. Toxicity of RB was mainly hematological with $29 \%$ rate of grade $3 / 4$ neutropenic events.

In the BRIGHT phase 3 randomized study, RB was compared with R-CHOP or R-CVP [15] for the initial treatment of patients with indolent and MCL. In this study BR therapy was non inferior to the standard therapy in terms of $C R$ rate $(31 \%$ and $25 \%$ in the $B R$ and in the standard-therapy treatment group, respectively) $(p=0,0225)$. Similar to what observed in the 
Stil study, toxicity of RB was mainly hematological with $39-49 \%$ of grade $3 / 4$ neutropenic events.

Finally, a prospective phase II trial has been carried out by the GELTAMO group [22] in untreated patients with CD20 MALT lymphoma requiring systemic therapy. Patients were treated with RB regimen; at the end of treatment, ORR was $100 \%$ with a CR rate of $98 \%$.

The rate of grade $3 / 4$ neutropenic event was $20 \%$ with a $5 \%$ rate of febrile neutropenia.

With the exception of the outstanding results of the GELTAMO trial, in our study we were able to confirm that activity of RB combination in INFL is high and can be set at $30-40 \%$ and at $80-90 \%$ when measured in terms of CR or of ORR, respectively. Differently from the other studies our series was characterized by the highest rates of severe neutropenia and by a high rate of G-CSF use increasing to a maximum rate of $48 \%$ at cycle 4 .

These rates are closer to the ones observed in patients with CLL [23] but cannot be clearly explained by the characteristics of our patients. Looking at the very low rate of infectious complication observed in our study and confirmed by the main published series, however, hematologic toxicity with RB is confirmed as highly manageable.

Finally in order to put our results in the right context major limitations of the study should be acknowledged. The major problem with our study is with the initial assumptions of a $75 \%$ CR rate with $\mathrm{RB}$ that is $15 \%$ increase from a $\mathrm{HO}$ of $60 \%$. When the study was designed data on the activity of RB were not fully available and considering the superiority versus R-CHOP observed in the Still trial it was hypothesized that similar improvement could be observed if FC-R was used as a comparator. $\mathrm{H} 0$ of $60 \%$ was defined using our previous experience with FCR on the same patient population [12]. With additional data from the Still study and with 
the Bright study results it was then clear that superiority of BR activity over the standards is not a realistic goal. If we had to design the INFL09 study today we would have been much more conservative modifying both $\mathrm{H} 0$ and $\mathrm{H} 1$ of the study.

Second, while, inclusion of different histological subtypes might be regarded as a shortcoming of the present study, it is to be underlined that such lymphoma entities, although different in terms of pathologic features, share a common clinical presentation, a similar overall prognosis and are currently managed with the same treatment approach. The relatively rare incidence of each distinctive INFL subtype and the lack of straightforward diagnostic criteria to define differential diagnosis makes it difficult to investigate each single lymphoma subtype separately also within large cooperative groups. Nonetheless some differences in terms of ORR and PFS were observed in our study, with relatively lower activity of RB in MZL compared with other subtypes, similar to what suggested by the Stil study (14); however, our data cannot be used to draw any conclusion on a different recommendation concerning the use of RB combination in INFL subtypes. Only studies specifically designed for specific INFL subtypes might clarify if a different activity of RB across INFL subtype is real.

In conclusion the INFLO9 results provide new data on the good activity and the good safety profile of RB combination for the treatment of indolent lymphomas and prompt this combination as a valuable option in patients with advanced INFL requiring treatment.

Several first- and second-generation small molecule inhibitors targeting multiple signalling pathways relevant to tumour cell growth are being extensively tested in indolent $\mathrm{NHL}$. These molecules, including $\mathrm{PI} 3 \mathrm{~K} / \mathrm{Akt} / \mathrm{mTOR}$, HDAC, BTK, proteasome inhibitors and immunomodulatory drugs, have shown promising results across a wide range of indolent 
lymphoma subtypes. However, these molecules usually display a moderate single-agent activity, as well as due to compensatory pathway activation and acquired resistance mechanisms, should be ideally combined with conventional anticancer drugs, to maximize their therapeutic potentials. Given its substantial anti-lymphoma activity and the absence of overlapping severe extra-haematological toxicities, the RB platform may represent a valuable candidate for combination with such newer target-based agents in non-follicular indolent lymphomas.

FUNDING SUPPORT

We gratefully acknowledge Mundipharma Pharmaceuticals srl Italia for providing a contribution consisting of drug supply (bendamustine) and a financial support to the study.

\section{AUTHORS' DISCLOSURES OF POTENTIAL CONFLICTS OF INTEREST}

Stefano Luminari has received personal fees from Pfizer and serves in an advisory role for Roche, Celgene and Teva outside the submitted work. Luca Arcaini has received personal fees from Mundipharma and serves in an advisory role for Roche, Celgene and Gilead outside the submitted work. Antonello Pinto has received personal fees from Roche, Celgene, Takeda and Mundipharma and has received non-financial support from Mundipharma, outside the submitted work.

All other author report no potential conflicts of interest.

\section{AUTHOR CONTRIBUTIONS}


SL was the principal investigator and takes primary responsibility for the paper. SL and LB designed the study. MG, SL, LO, AT, AP, FS, LA, AMC, AT, AP, CT and LB recruited the patients. MC coordinated patient's recruitment and was in charge of data quality check. SL and LB co-ordinated the research. SL, MC and LB wrote the paper. All authors have critically reviewed and approved the final manuscript.

\section{REFERENCES}

1. Swerdlow SH, Campo E, Harris NL, et al. World Health Organization classification of tumors of hematopoietic and lymphoid tissues. 4th ed. Lyon, IARC, 2008.

2. Ben-Ezra J, Burke JS, Swartz WG, et al. Small lymphocytic lymphoma: a clinicopathologic analysis of 268 cases. Blood. 1989;73(2):579-587.

3. Coiffier B, Thieblemont C, Felman $\mathrm{P}$, el al. Indolent non follicular lymphomas: characteristics, treatment, and outcome. Semin Hematol. 1999;36(2):198-208. 
4. Landgren O, Tilly H. Epidemiology, pathology and treatment of non-follicular indolent lymphomas. Leuk Lymphoma. 2008;49(1 Suppl):35-42.

5. Pangalis GA, Angelopoulou MK, Vassilakopoulos TP, et al. B-chronic lymphocytic leukemia, small lymphocytic lymphoma, and lymphoplasmacytic lymphoma, including Waldenstrom's macroglobulinemia: a clinical, morphologic, and biologic spectrum of similar disorders. Semin Hematol. 1999;36(2):104-114.

6. Papamichael D, Norton AJ, Foran JM, et al. Immunocytoma: a retrospective analysis from St Bartholomew's Hospital-1972 to 1996. J Clin Oncol. 1999;17(9):2847-2853.

7. Seng JE, Peterson BA. Indolent B-cell non-Hodgkin's lymphomas. Oncology (Williston Park). 1997;11(12):1883-1894.

8. Neparidze N, Dhodapkar MV. Waldenstrom's macroglobulinemia: Recent advances in biology and therapy. Clin Adv Hematol Oncol. 2009;7(10):677-690.

9. Treon SP. How I treat Waldenstrom macroglobulinemia. Blood. 2009;114(12): 23752385.

10. Zinzani PL. Traditional treatment approaches in B-cell non-Hodgkin's lymphoma. Leuk Lymphoma. 2003;44(4 Suppl):S6-14.

11. Baldini L, Brugiatelli M, Luminari S, et al. Treatment of indolent B-Cell non follicular lymphomas: final results of the LL01 randomized trial of the Gruppo Italiano per lo Studio dei Linfomi. J Clin Oncol. 2003;21(8):1459-1465.

12. Ferrario A, Pulsoni A, Olivero B, et al. Fludarabine, cyclophosphamide, and rituximab in Patients With Advanced, Untreated, Indolent B-Cell Non follicular Lymphomas: phase 2 
study of the Italian Lymphoma Foundation. Cancer 2012;118(16):3954-61.

13. Ferrario A, Merli F, Luminari S, et al. Phase II fludarabine and cyclophosphamide for

the treatment of indolent B cell non-follicular lymphomas: final results of the LLO2 trial of the Gruppo Italiano per lo Studio dei Linfomi (GISL). Ann Hematol. 2011;90(3):323-30.

14. Rummel MJ, Niederle N, Maschmeyer G, et al; Study group indolent Lymphomas (StiL). Bendamustine plus rituximab versus $\mathrm{CHOP}$ plus rituximab as fi rst-line treatment for patients with indolent and mantle-cell lymphomas: an open-label, multicentre, randomised, phase 3 non-inferiority trial. Lancet 2013;381(9873):1203-10.

15. Flinn IW, van der Jagt R, Kahl BS, et al. Randomized trial of bendamustine-rituximab or R-CHOP/R-CVP in first-line treatment of indolent NHL or MCL: the BRIGHT study. Blood 2014; 123(19):2944-52.

16. Hryniuk WM: Average relative dose intensity and the impact on design of clinical trials. Semin Oncol. 1987;14:65-74.

17. Cheson BD, Pfistner B, Juweid ME, et al. Revised Response Criteria for Malignant Lymphoma. J Clin Oncol. 2007;25(5):579-86.

18. Rummel MJ, Al-Batran SE, Kim SZ et al. Bendamustine plus rituximab is effective and has a favorable toxicity profile in the treatment of mantle cell and low-grade non-hodgkin's Lymphoma. J Clin Oncol. 2005;23(15):3383-9.

19. Hallek M, Fischer K, Fingerle-Rowson G, et al. Addition of rituximab to fludarabine and cyclophosphamide in patients with chronic lymphocytic leukaemia: a randomised, openlabel, phase 3 trial. Lancet. 2010;376:1164-1174. 
20. Sacchi S, Marcheselli L, Bari A, et al. Secondary malignancies after treatment for indolent non-Hodgkin's lymphoma: a 16-year follow-up study. Haematologica, 2008 Mar;93(3):398-404.

21. Waterman J, Rybicki L, Bolwell B, et al. Fludarabine as a risk factor for poor stem cell harvest, treatment-related MDS and AML in follicular lymphoma patients after autologous hematopoietic cell transplantation. Bone Marrow Transplant. 2012 Apr;47(4):488-93.

22. Salar A, Domingo-Domenech E, Panizo C, et al. First-line response-adapted treatment with the combination of bendamustine and rituximab in patients with mucosa-associated lymphoid tissue lymphoma (MALT2008-01): a multicentre, single-arm, phase 2 trial. Lancet Haematology. 2014 Dec;1(3):e104-e111.

23. Eichhorst B, Fink A.M, Busch R, et al. Frontline Chemoimmunotherapy with Fludarabine (F), Cyclophosphamide (C), and Rituximab (R) (FCR) Shows Superior Efficacy in Comparison to Bendamustine (B) and Rituximab (BR) in Previously Untreated and Physically Fit Patients (pts) with Advanced Chronic Lymphocytic Leukemia (CLL): Final Analysis of an International, Randomized Study of the German CLL Study Group (GCLLSG) (CLL10 Study). Oral presentation at the 56th American Society of Hematology (ASH) Annual Meeting in San Francisco - abstract number 19 session 642.

FIGURE LEGENDS

Figure 1. Reason for treatment interruption by cycle in patients who prematurely discontinued RB protocol. 


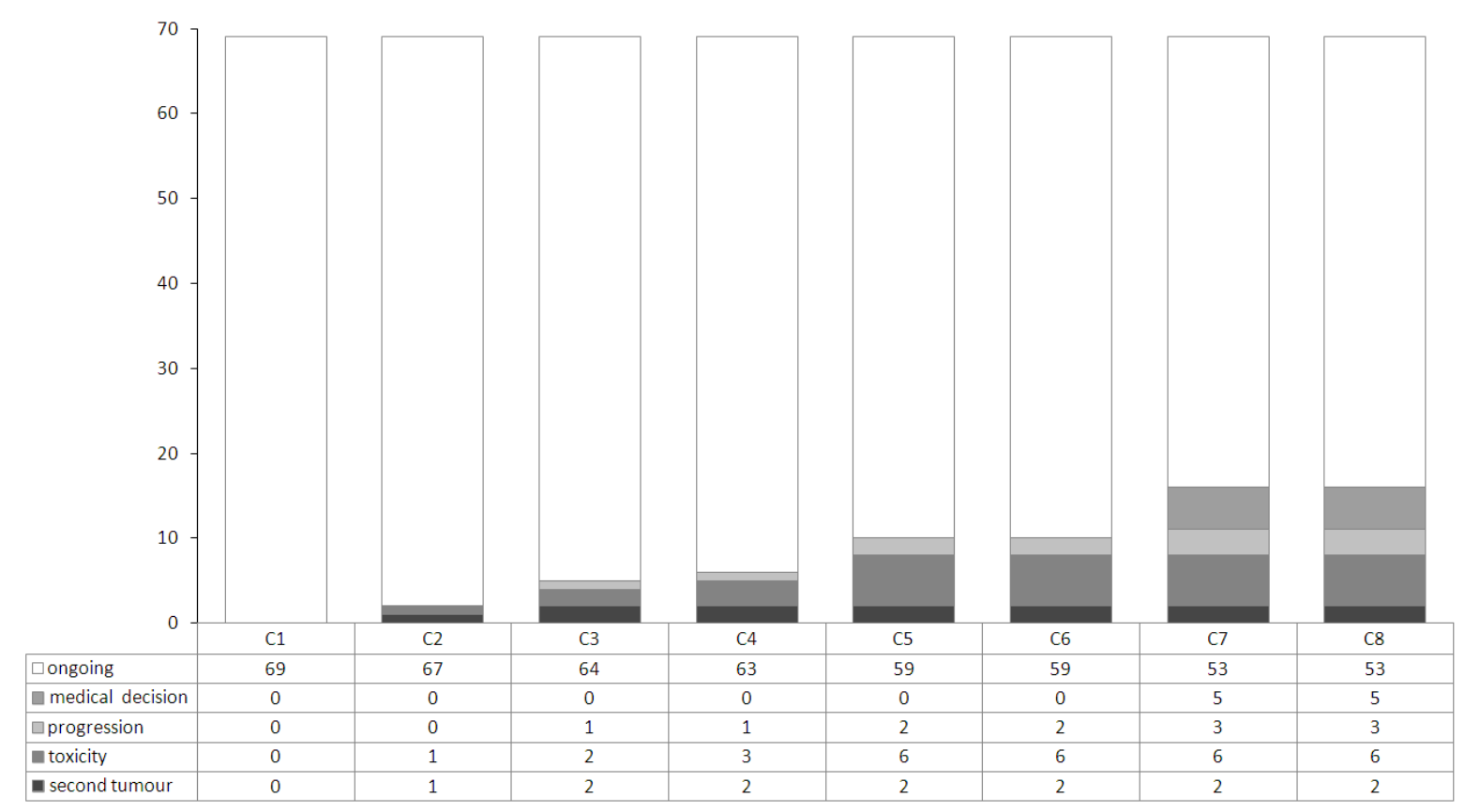

Figure 1. Reason for treatment interruption by cycle in patients who prematurely discontinued RB protocol.

Figure 2. OS (a) and PFS (b) of 69 eligible patients 

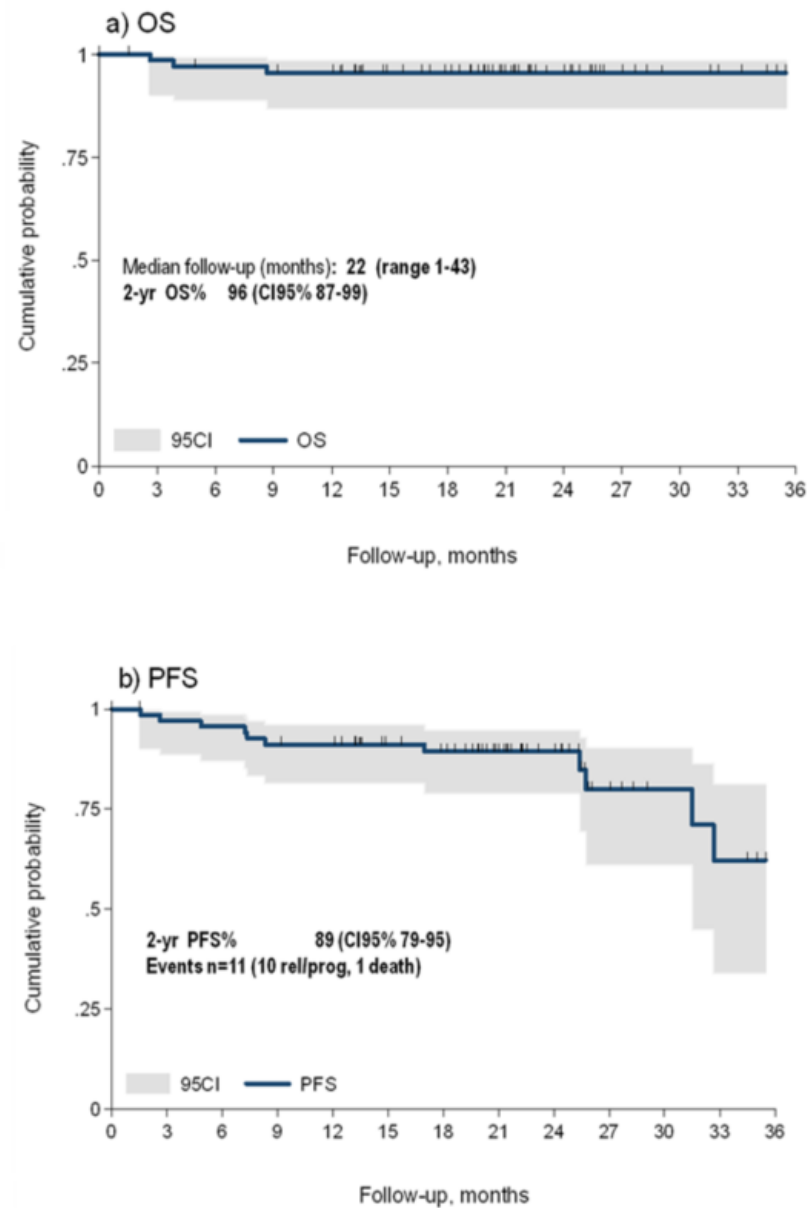

Figure 2. OS (a) and PFS (b) of 69 eligible patients 
Table I. Patient characteristics $(\mathrm{N}=69)$

\begin{tabular}{|c|c|c|}
\hline Characteristics & $\mathbf{N}$ & $\%$ \\
\hline $\mathrm{M} / \mathrm{F}$ & \multicolumn{2}{|c|}{$45(65 \%) / 24(35 \%)$} \\
\hline Median age & \multicolumn{2}{|c|}{$65(45-75)$} \\
\hline LPL/IC & 30 & 43 \\
\hline MZL & 18 & 26 \\
\hline SLL & 13 & 19 \\
\hline LG NOS & 8 & 12 \\
\hline III-IV stage & 68 & 99 \\
\hline B symptoms & 11 & 16 \\
\hline ECOG PS & & \\
\hline $0-1$ & 64 & 93 \\
\hline $2-3$ & 5 & 7 \\
\hline LDH $>$ UNL & 10 & 14 \\
\hline B-2-micro>UNL & 43 & 62 \\
\hline Median hb & \multicolumn{2}{|c|}{$11.60(4.6-15.7)$} \\
\hline Median PLT & \multicolumn{2}{|c|}{194 (36-499) } \\
\hline Median lymphocyte & \multicolumn{2}{|c|}{$1.80(0.3-71.2)$} \\
\hline $\mathrm{BM}+$ & 64 & 93 \\
\hline Serum MC & 45 & 66 \\
\hline
\end{tabular}




\begin{tabular}{|c|c|c|}
\hline FLIP & 3 & 4 \\
$0-1$ & 19 & 28 \\
2 & 47 & 68 \\
\hline
\end{tabular}

Abbreviations: LPL/IC denotes lymphoplasmacytic lymphomas, MZL denotes marginal zone lymphomas, SLL denotes small lymphocytic lymphoma, LG NOS denotes low grade not otherwise specified, ECOG denotes eastern cooperative oncology group, PS denotes performance status, LDH denotes Lactate dehydrogenase, UNL denotes upper normal limit, hb denotes hemoglobin, PLT denotes platelet, BM denotes bone marrow, MC denotes monoclonal antibody, FLIPI: follicular lymphoma international prognostic index.

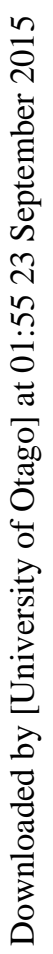

26 
Table II. Centrally reviewed response by INFL subtype*.

\begin{tabular}{|c|c|c|c|c|c|c|c|c|c|c|}
\hline & \multicolumn{2}{|c|}{ ALL CASES (N=69) } & \multicolumn{2}{c|}{ MZL (N=18) } & \multicolumn{2}{c|}{ LPL/IC (N=30) } & \multicolumn{2}{c|}{ SLL (N=13) } & \multicolumn{2}{c|}{ LG NOS (N=8) } \\
\cline { 2 - 11 } & $\mathbf{N}$ & $\mathbf{\%}$ & $\mathbf{N}$ & $\mathbf{\%}$ & $\mathbf{N}$ & $\mathbf{\%}$ & $\mathbf{N}$ & $\mathbf{\%}$ & $\mathbf{N}$ & $\mathbf{\%}$ \\
\hline CR & 33 & 48 & 10 & 56 & 13 & 43 & 8 & 62 & 2 & 25 \\
\hline PR & 26 & 38 & 3 & 17 & 16 & 53 & 4 & 31 & 3 & 38 \\
\hline SD & 0 & 0 & 0 & 0 & 0 & 0 & 0 & 0 & 0 & 0 \\
\hline PD & 6 & 9 & 2 & 11 & 1 & 3 & 1 & 8 & 2 & 25 \\
\hline NE & 4 & 6 & 3 & 17 & 0 & 0 & 0 & 0 & 1 & 13 \\
\hline
\end{tabular}

ORR

\begin{tabular}{l|l}
59 & 86
\end{tabular}

13

72

96

12

92

5

*INFL subtype was reclassified after review of pathology reports.

Abbreviations: CR denotes complete remission, PR denotes partial remission, SD denotes stable disease, PD denotes progressive disease, NE denotes not evaluable, ORR denotes overall response rate, LPL/IC denotes lymphoplasmacytic lymphomas, , SLL denotes small lymphocytic lymphoma, MZL denotes marginal zone lymphomas, LG NOS denotes low grade not otherwise specified. 
Table III. Summary of grade 3/4 adverse events reported in the trial and of grade $1 / 2$ events reported in more than one patient $\left({ }^{*}\right)$.

\begin{tabular}{|l|c|c|c|c|}
\hline $\begin{array}{c}\text { HEMATOLOGICAL TOXICITY } \\
\text { (N=69) }\end{array}$ & $\mathbf{1 / 2}$ & $\mathbf{\%}$ & $\mathbf{3 / 4}$ & $\mathbf{\%}$ \\
\hline Anemia & 27 & 39 & 3 & 4 \\
\hline Leucopenia & 13 & 19 & 21 & 30 \\
\hline Neutropenia & 14 & 20 & 30 & 43 \\
\hline Thrombocytopenia & 16 & 23 & $5^{* *}$ & 7 \\
\hline Febrile Neutropenia & - & - & 2 & 3 \\
\hline
\end{tabular}

** In 5 patients thrombocytopenia was present before treatment start and only 1 patient developed severe thrombocytopenia during therapy

\begin{tabular}{|l|c|c|c|c|}
\hline \multicolumn{1}{|c|}{$\begin{array}{c}\text { NON HEMATOLOGICAL TOXICITY } \\
\text { (N=69) }\end{array}$} & $\mathbf{1 / 2}$ & $\%$ & $3 / 4$ & $\%$ \\
\hline NEUROLOGY & 1 & 1 & 1 & 1 \\
\hline Syncope & 5 & 7 & - & - \\
\hline MUSCULOSKELETAL/SOFT TISSUE & 6 & 9 & 1 & 1 \\
\hline Bone pain / pain & 2 & 3 & - & - \\
\hline Muscle weakness & - & - & 1 & 1 \\
\hline CONSTITUTIONAL SYMPTOMS & 14 & 20 & - & - \\
\hline Chills & 9 & 13 & - & - \\
\hline Weight loss & & & \\
\hline Fever & & & & \\
\hline GASTROINTESTINAL & & & \\
\hline Constipation
\end{tabular}




\begin{tabular}{|c|c|c|c|c|}
\hline Anorexia & 3 & 4 & - & - \\
\hline Diarrhea & 4 & 6 & - & - \\
\hline Taste alteration (dysgeusia) & 3 & 4 & - & - \\
\hline Heartburn/dyspepsia & 2 & 3 & - & - \\
\hline Mucositis oral / Mucositis & 3 & 4 & - & - \\
\hline Nausea & 15 & 22 & 2 & 3 \\
\hline Vomiting & 6 & 9 & 1 & 1 \\
\hline \multicolumn{5}{|l|}{ PULMONARY/UPPER RESPIRATORY } \\
\hline Cough & 2 & 3 & - & - \\
\hline Pneumonia/respiratory infection & - & - & 1 & 1 \\
\hline \multicolumn{5}{|l|}{ INFECTION } \\
\hline Infection & 9 & 13 & 1 & 1 \\
\hline \multicolumn{5}{|l|}{ CARDIAC GENERAL } \\
\hline Myocarditis & - & - & 1 & 1 \\
\hline \multicolumn{5}{|l|}{ DERMATOLOGY/SKIN } \\
\hline Rash & 12 & 17 & 2 & 3 \\
\hline \multicolumn{5}{|l|}{ SECONDARY MALIGNANCY } \\
\hline Secondary maglinancies & - & - & 2 & 3 \\
\hline \multicolumn{5}{|l|}{ LYMPHATICS } \\
\hline Edema: limb & 2 & 3 & - & - \\
\hline \multicolumn{5}{|l|}{ VASCULAR } \\
\hline Thrombosis/thrombus/embolism & 1 & 1 & 1 & 1 \\
\hline \multicolumn{5}{|l|}{ ALLERGY/IMMUNOLOGY } \\
\hline Allergic reaction/hypersensitivity & 1 & 1 & 1 & 1 \\
\hline
\end{tabular}


* Adverse event categories with only one grade 1-2 event and no grade 3-4 were not included in the table 